\title{
Caffeine prevents human prion protein-mediated neurotoxicity through the induction of autophagy
}

\author{
JI-HONG MOON ${ }^{1}$, JU-HEE LEE ${ }^{1}$, JIN-YOUNG PARK ${ }^{1}$, SUNG-WOOK KIM ${ }^{1}$, YOU-JIN LEE ${ }^{1}$, \\ SEOG-JIN KANG ${ }^{2}$, JAE-WON SEOL ${ }^{1}$, DONG-CHOON AHN ${ }^{1}$ and SANG-YOUEL PARK ${ }^{1}$ \\ ${ }^{1}$ Biosafety Research Institute, College of Veterinary Medicine, Chonbuk National University, Jeonju, Jeonbuk 561-756; \\ ${ }^{2}$ National Institute of Animal Science, Rural Development Administration, Cheonan, Chungnam 330-801, Republic of Korea
}

Received January 23, 2014; Accepted June 16, 2014

DOI: $10.3892 / \mathrm{ijmm} .2014 .1814$

\begin{abstract}
The human prion protein (PrP) fragment PrP(106-126) possesses the majority of the pathogenic properties associated with the infectious scrapie isoform of PrP, known as $\mathrm{PrP}^{\mathrm{Sc}}$. The accumulation of $\mathrm{PrP}^{\mathrm{Sc}}$ in the brain of humans and animals affects the central nervous system. Recent epidemiological studies have suggested that caffeine, one of the major components of coffee, exerts protective effects against the development of neurodegeneration. However, the protective effects of caffeine against prion disease have not been reported to date. In this study, we therefore investigated the effects of caffeine on PrP-mediated neurotoxicity. The protein expression of the autophagosomal marker, LC3-II, was increased by caffeine in a dose-dependent manner, and the autophagy induced by caffeine protected the neuronal cells against $\operatorname{PrP}(106-126)$-induced cell death. On the contrary, the downregulation of LC3-II using the autophagy inhibitors, 3-methyladenine (3-MA) and wortmannin, prevented the caffeine-mediated neuroprotective effects. To the best of our knowledge, the present study provides the first evidence that treatment with caffeine protects human neuronal cells against prion-mediated neurotoxicity and these neuroprotective effects are mediated by caffeine-induced autophagy signals. Our data suggest that treatment with caffeine may be a novel therapeutic strategy for prion peptide-induced apoptosis.
\end{abstract}

\section{Introduction}

The misfolding and aggregation of specific proteins is a common hallmark of a number of neurodegenerative disorders, including highly prevalent illnesses, such as Alzheimer's and Parkinson's disease, as well as more rare disorders, such as

Correspondence to: Professor Sang-Youel Park or Dr Dong-Choon Ahn, Biosafety Research Institute, College of Veterinary Medicine, Chonbuk National University, 567 Baekje-daero, Deokjin-gu, Jeonju, Jeonbuk 561-756, Republic of Korea

E-mail: sypark@chonbuk.ac.kr

E-mail: ahndc@chonbuk.ac.kr

Key words: caffeine, $\operatorname{PrP}(106-126)$, autophagy, neuroprotection prion diseases (1). Prion diseases, also known as transmissible spongiform encephalopathies (TSEs), are a group of fatal neurodegenerative disorders caused by the misfolding of normal cellular prion $\left(\mathrm{PrP}^{\mathrm{C}}\right)$ into an abnormal form of scrapie isoform of prion protein $\left(\mathrm{PrP}^{\mathrm{Sc}}\right)$ (2). The basic mechanism of prion infectivity is the ability to self-reproduce through the conversion of $\mathrm{PrP}^{\mathrm{C}}$ into $\mathrm{PrP}^{\mathrm{Sc}}$, a transformed protease-resistant protein that is believed to be the infectious agent in the transmissible form of prion disease (3). Although it is well established that $\mathrm{PrP}^{\mathrm{Sc}}$ plays a major role in the origin and transmission of TSEs, the mechanisms through which the disease-specific PrP exerts its harmful effects on neurons remain unknown (4).

$\operatorname{PrP}(106-126)$, a peptide fragment of $\operatorname{PrP}^{\mathrm{Sc}}$, was used in this study for the experimental induction of $\operatorname{PrP}^{\mathrm{sc}}$ infection. $\operatorname{PrP}(106-$ 126) possesses several of the pathogenic and physiological properties of $\mathrm{PrP}^{\mathrm{Sc}}$, including the ability to induce apoptosis in hippocampal neurons and to induce the proliferation of astrocytes (5). It also catalyzes the aggregation of endogenous cellular $\operatorname{Pr} P\left(\operatorname{PrP}^{\mathrm{C}}\right)$ into an amyloidogenic form that shares several characteristics with $\operatorname{PrP}^{\mathrm{Sc}}$ (6). Therefore, this peptide is useful for in vitro studies of prion-induced neuronal apoptosis.

The term 'autophagy', which means 'eating of self', was first coined by Deter and De Duve several decades ago and was primarily based on the observed degradation of the mitochondria and cellular components within lysosomes (7). Autophagy encompasses the different routes that cells use to deliver cytoplasmic substrates to lysosomes for degradation (8). There are three defined types of autophagy: macroautophagy, microautophagy and chaperone-mediated autophagy (CMA), all of which promote the proteolytic degradation of the intracellular components at the lysosome (9). In the present study, we focused on macroautophagy. Upon induction, the phagophore expands and encloses a portion of the cytoplasm, resulting in the formation of a double-membraned structure termed the autophagosome, which fuses with a lysosome for degradation (10). Autophagy, a common morphological feature in dying cells, was often erroneously presumed to be a cell death pathway. However, it now seems clear that one of its major functions is to keep cells alive under stressful 'lifethreatening' conditions (11). The suppression of autophagy is associated with certain diseases, including a subset of cancers, neurodegenerative disorders, infectious diseases and inflammatory bowel disorders, and a decline in autophagy function 
is a common feature of aging (10). The neuroprotective effects mediated by the inducers of autophagy are related to an increase in the mitochondrial turnover as a result of autophagy activation $(12,13)$. In addition, autophagy reduces the amount of abnormal PrP (14).

Caffeine is a widely used psychoactive drug that has been long used to increase alertness and energy. It is also known to exert neuroprotective effects against Parkinson's disease, one of the many neurodegenerative disorders (15). However, little is known about the effects of caffeine on autophagy and cellular homeostasis in prion diseases.

In this study, we investigated the protective effects of caffeine against the neurotoxicity that is associated with prions using the $\operatorname{PrP}(106-126)$ peptide in a SH-SY5Y neuroblastoma cell line. Our results demonstrate that caffeine enhances autophagy in a dose-dependent manner in neuroblastoma cells. Furthermore, we reveal that caffeine-induced autophagy protects these cells against $\operatorname{PrP}(106-126)$-induced apoptosis.

\section{Materials and methods}

Cell culture. The human neuroblastoma cell line, SH-SY5Y, was obtained from the American Type Culture Collection (ATCC; Rockville, MD, USA). The cells were cultured in minimum essential medium (Invitrogen-Gibco, Carlsbad, CA, USA) supplemented with $10 \%$ fetal bovine serum (Invitrogen-Gibco), $100 \mathrm{U} / \mathrm{ml}$ penicillin and $0.1 \mathrm{mg} / \mathrm{ml}$ gentamycin in a humidified incubator maintained at $37^{\circ} \mathrm{C}$ and $5 \% \mathrm{CO}_{2}$. The cells were treated with various doses of caffeine (Sigma-Aldrich, St. Louis, MO, USA) for $1 \mathrm{~h}$ and were then exposed to $50 \mu \mathrm{M}$ $\operatorname{PrP}(106-126)$ for $24 \mathrm{~h}$ with or without the autophagy inhibitors, 3-methyladenine (3-MA; $200 \mu \mathrm{M})$ and wortmannin $(50 \mathrm{nM})$ (Sigma-Aldrich).

Treatment with $\operatorname{Pr} P(106-126)$. Synthetic $\operatorname{PrP}(106-126)$ peptides (sequence, Lys-Thr-Asn-Met-Lys-His-Met-Ala-Gly-Ala-AlaAla-Ala-Gly-Ala-Val-Val-Gly-Gly-Leu-Gly) were synthesized by Peptron (Seoul, Korea). The peptides were dissolved in sterile dimethyl sulfoxide at a stock concentration of $10 \mathrm{mM}$ and stored at $-4^{\circ} \mathrm{C}$.

Terminal deoxynucleotidyl transferase dUTP nick end-labeling (TUNEL) assay. TUNEL assay was performed to measure the degree of cellular apoptosis using an In Situ ApoBrdU DNA fragmentation assay kit (BioVision, Mountain View, CA, USA), according to the manufacturer's instructions. The cells were counterstained with propidium iodide (PI) to show cell nuclei.

Annexin V assay. Apoptosis in the detached cells was assessed using an Annexin V assay kit (Santa Cruz Biotechnology, Santa Cruz, CA, USA) according to the manufacturer's instructions. The Annexin V levels were determined by measuring fluorescence at an excitation of $488 \mathrm{~nm}$ and an emission of 525/30 using a Guava EasyCyte HT System (Millipore, Bedford, MA, USA).

Trypan blue exclusion assay. The number of viable cells was determined by trypan blue dye exclusion (Sigma-Aldrich) using a hemocytometer. The result was expressed as a percentage relative to the control.
Western blot analysis. The SH-SY5Y cells were lysed in lysis buffer [25 mM 4-(2-hydroxyethyl)-1-piperazineethanesulfonic acid (HEPES), pH 7.4, $100 \mathrm{mM} \mathrm{NaCl}, 1 \mathrm{mM}$ ethylenediaminetetraacetic acid (EDTA), $5 \mathrm{mM} \mathrm{MgCl} 2,0.1 \mathrm{mM}$ dithiothreitol (DTT), and a protease inhibitor mixture] and whole cell proteins were electrophoretically resolved on a $10-15 \%$ sodium dodecyl sulfate polyacrylamide gel and transferred onto a nitrocellulose membrane. Immunoreactivity was detected through sequential incubation with primary antibodies, horseradish peroxidase-conjugated secondary antibodies and enhanced chemiluminescence reagents using the horseradish peroxidase detection kit (Westsave Gold;,AbFrontier Inc., Seoul, Korea). The primary antibodies used for immunoblotting were as follows: anti-LC3 (Novus Biologicals, Littleton, CO, USA), antiphospho-SAPK/JNK (Cell Signaling Technology Inc., Danvers, MA, USA), anti-phospho-Akt (Epitomics, Burlingame, CA, USA) and anti- $\beta$-actin (Sigma-Aldrich). Images were captured using a Fusion FX7 imaging system (Vilber Lourmat, ZI Sud Torcy, France). The densitometry of the signal bands was analyzed using Bio-1D software (Vilber Lourmat, Marne la Vallée, France).

\section{Results}

Caffeine inhibits PrP(106-126)-induced apoptosis. We examined whether caffeine protects neuronal cells against prion-mediated neurotoxicity and whether these effects are associated with the induction of autophagy. First, we investigated the effects of caffeine on $\operatorname{PrP}(106-126)$-mediated neurotoxicity in the SH-SY5Y cells using an Annexin V assay. The SH-SY5Y cells were exposed to caffeine with or without $\operatorname{PrP}(106-126)$. The cell viability of the $\operatorname{PrP}(106-126)$-treated cells was decreased by approximately $50 \%$ compared with the untreated controls. The cell viability of the cells treated with caffeine only was comparable to that of the untreated controls. Importantly, treatment with caffeine inhibited $\operatorname{PrP}(106-126)$ induced neurotoxicity in the SH-SY5Y cells (Fig. 1A and B). We also used a TUNEL assay (Fig. 1C), microscopic imaging (Fig. 1D) and a trypan blue exclusion assay (Fig. 1E). As shown in Fig. 1C, the apoptotic process in the $\operatorname{PrP}(106-126)$ treated cells emitted green fluorescence, indicating DNA strand breakage. As shown in Fig. 1E, treatment with caffeine reversed the cell death induced by treatment with $\operatorname{PrP}(106-126)$ in a dose-dependent manner. These results indicated that caffeine was effective in preventing $\operatorname{PrP}(106-126)$-induced apoptosis in the SH-SY5Y cells.

Caffeine induces the production of the autophagosomal marker, LC3-II. The LC3 protein is localized and aggregated on the autophagosome and is therefore considered to be a marker for autophagy. LC3 transforms from LC3-I into LC3-II during autophagosome formation (16). Thus, in the present study, we investigated whether caffeine induces autophagy by measuring LC3 transformation. As shown in Fig. 2, we found that the levels of the late autophagosomal marker, LC3-II, increased in the caffeine-treated group in a dose-dependent manner compared with the control, as shown by western blot and densitometric analyses (Fig. 2A and B). These results suggested that caffeine induced autophagy in a dose-dependent manner. 
A
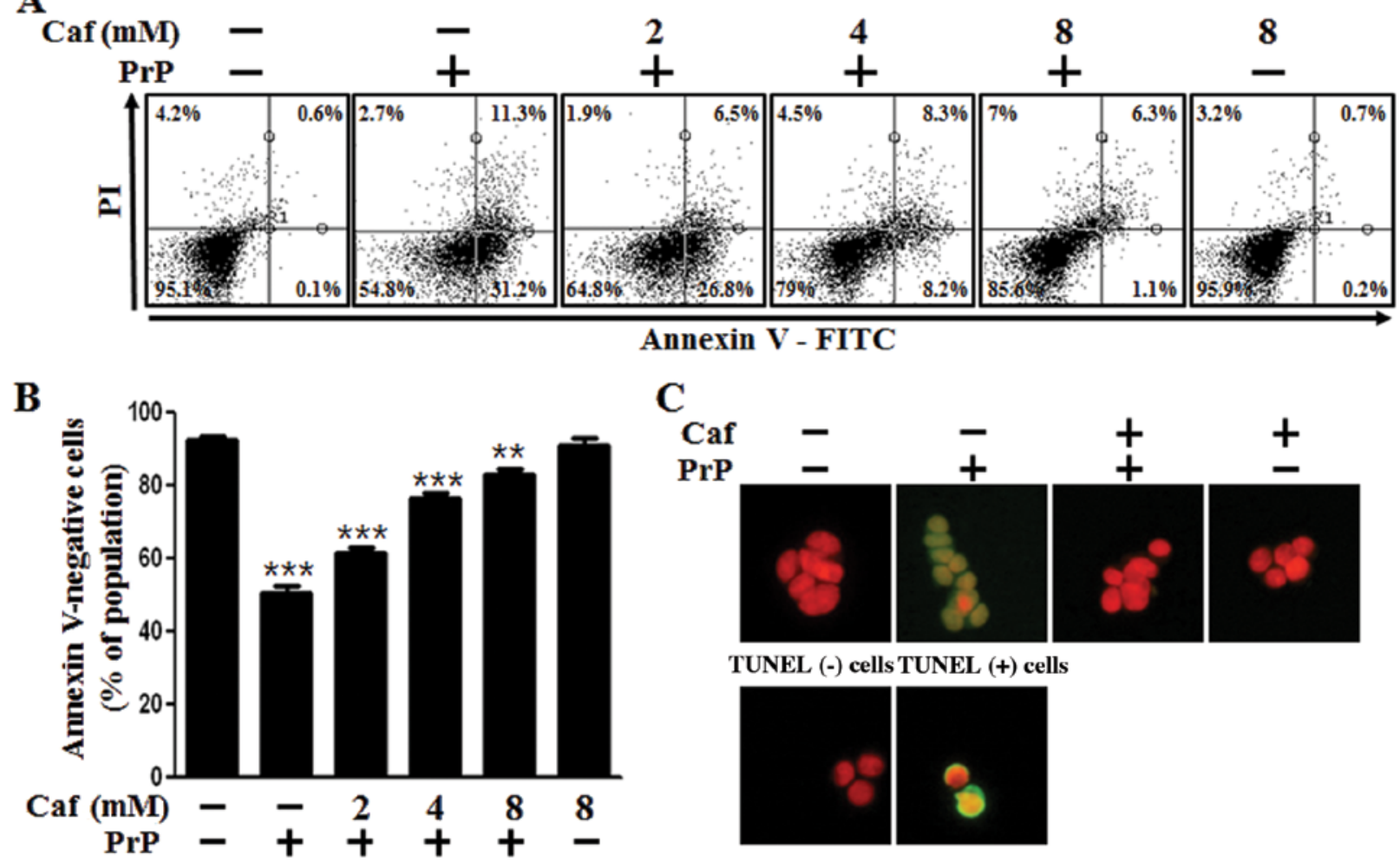

Annexin V - FITC

B

\section{D}

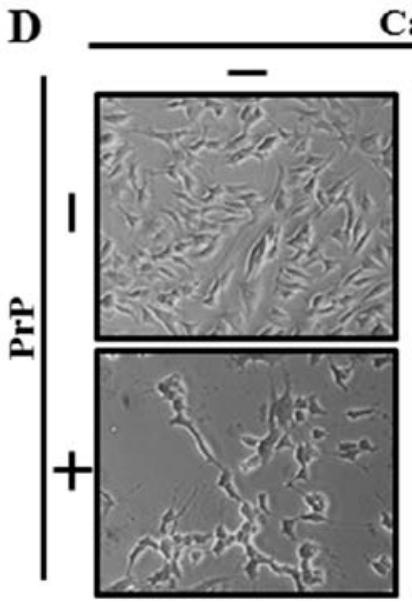

Caf

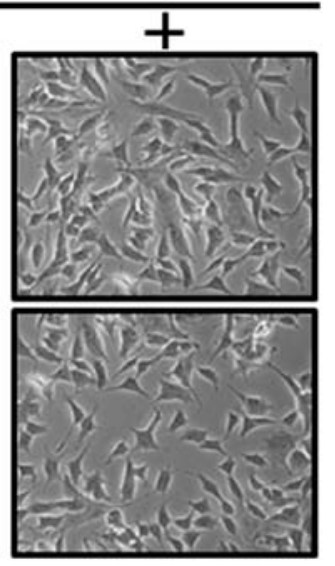

$\mathrm{C}$
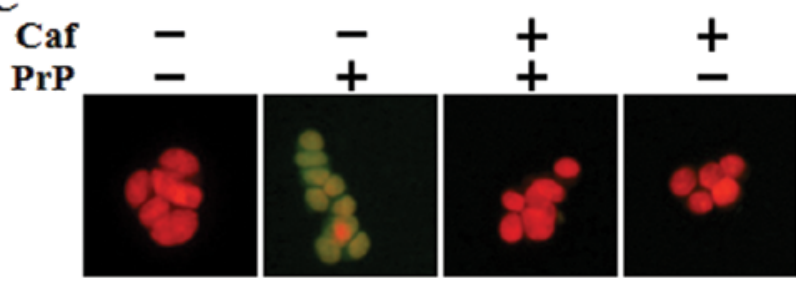

TUNEL (-) cells TUNEL (+) cells

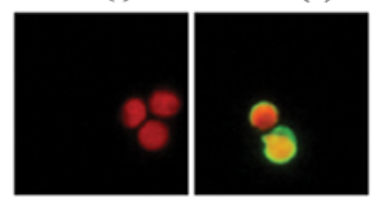

$\mathbf{E}$

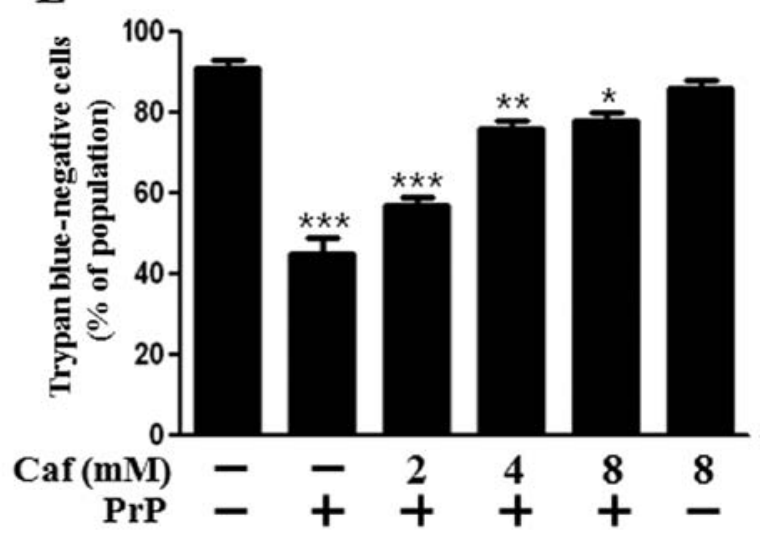

Figure 1. Treatment with caffeine inhibits PrP(106-126)-induced apoptosis. (A) SH-SY5Y neuronal cells were pre-treated with various doses of caffeine (1 h) and were then exposed to $50 \mu \mathrm{M} \operatorname{PrP}(106-126)$ for $24 \mathrm{~h}$. Cell viability was measured using an Annexin V assay. Cells were treated with FITC-Annexin V, which binds to phosphatidylserine at the plasma membrane during apoptosis. (B) Bar graph indicates the averages of Annexin V-positive cells. (C) Representative immunofluorescence images of TUNEL-positive (green) SH-SY5Y cells at $24 \mathrm{~h}$ after exposure to 50 $\mu \mathrm{M}$ of PrP(106-126) in the absence or presence of caffeine $(1 \mathrm{~h})$. The cells were counterstained with propidium iodide (PI) (red) to show all cell nuclei. (D) The treated cells were also photographed with a light microscope (magnification, $\mathrm{x} 100$ ) and (E) analyzed using a trypan blue dye exclusion assay. ${ }^{*} \mathrm{P}<0.05,{ }^{* *} \mathrm{P}<0.01$ and ${ }^{* * *} \mathrm{P}<0.001$ : significant differences compared with the control and each treatment group. Caf, caffeine.

Caffeine inhibits $\operatorname{PrP}(106-126)$-induced apoptosis by activating autophagy. Accumulating evidence suggests a neuroprotective role of autophagy in the control of cell death (17-19). In this study, to investigate the effects of caffeine on the activation of autophagy, we analyzed the levels of LC3-II and cell viability using autophagy inhibitors. We found that the caffeine-induced increase in the LC3-II levels was inhibited by the autophagy inhibitors, 3-MA and wortmannin, as shown by western blot and densitometryic analyses (Fig. 3A and B). We also found that the effects of caffeine on cell viability were reversed by treatment with 3-MA and wortmannin, as shown by an Annexin-V assay and graph analysis (Fig. 3C and D). In Fig. 3C, which shows the results procuced by the Annexin V assay, the dots represent fluorescent cells in the plot. Green fluorescence (x-axis) indicates apoptosis and red fluorescence (y-axis) indicates necrosis. Cells in the first quadrant signify the late apoptotic condition and cells in the fourth quadrant signify the early apoptotic condition. We found that recovery in cell viability induced by caffeine was reversed by treatment with 3-MA and wortmannin. These results strongly suggest that caffeine exerts neuroprotective effects through the induction of autophagy. 
A

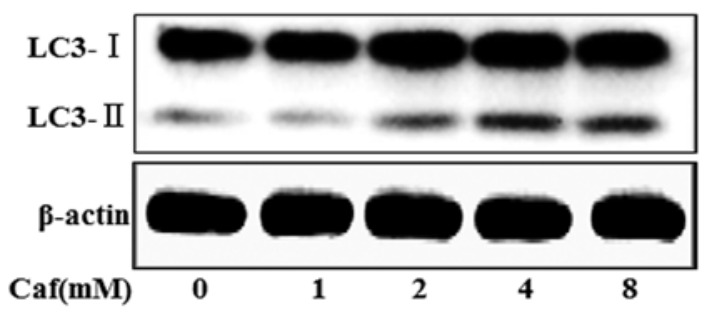

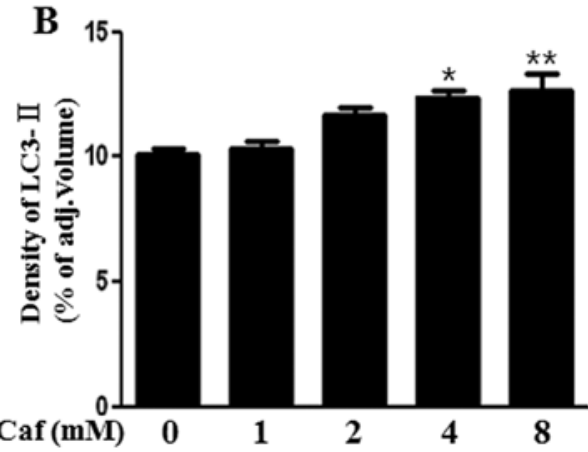

Figure 2. The administration of caffeine induces the production of the autophagosomal marker, LC3-II. (A) SH-SY5Y cells were treated with various doses of caffeine. The treated cells were assessed for LC3 production by western blot analysis. Results were normalized to $\beta$-actin. (B) The bar graph indicates the average of the LC3-II activity ratio. " $\mathrm{P}<0.05$ and ${ }^{* *} \mathrm{P}<0.01$ : significant differences between the control cells and each treatment group. Caf, caffeine; adj.volume, adjustment of volume (band volume minus background volume).

$\mathbf{A}$

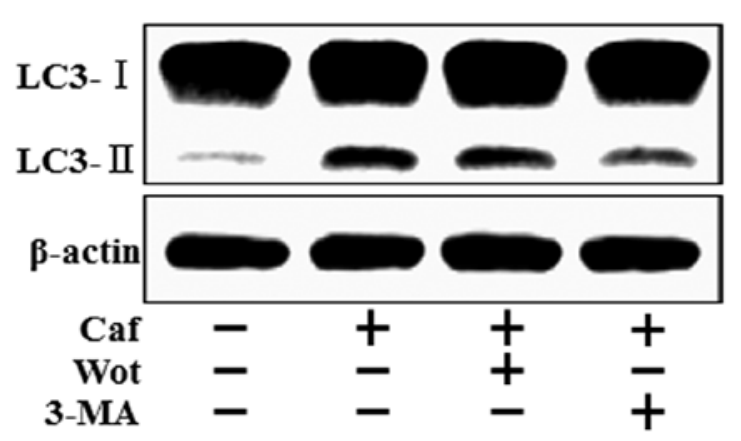

B

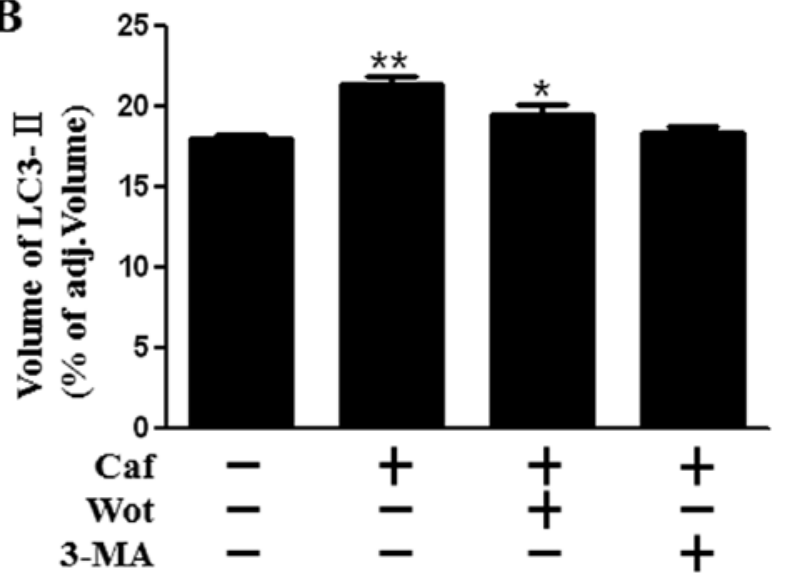

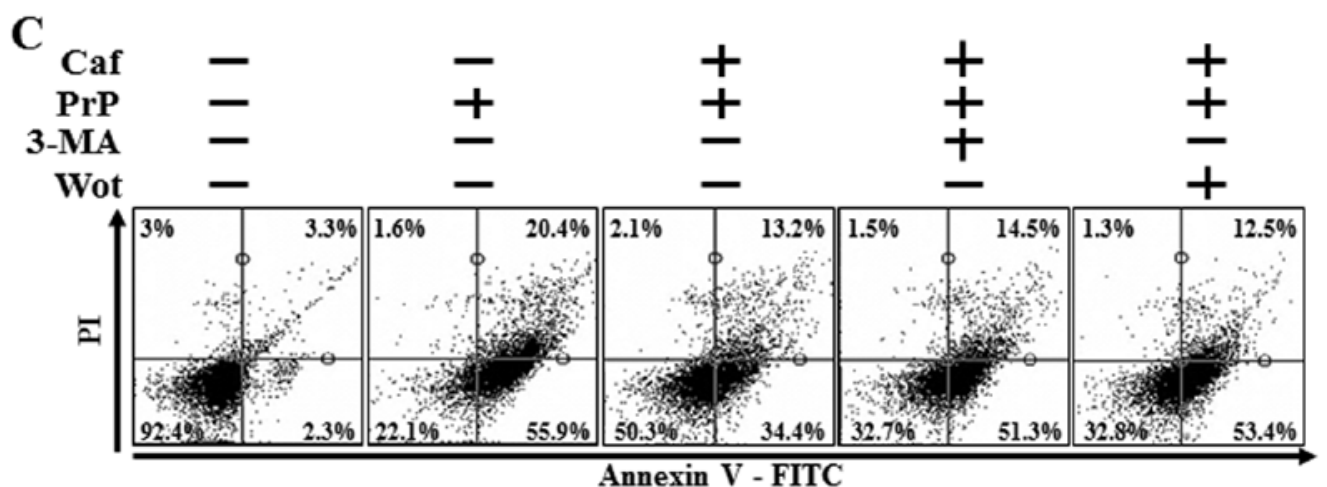

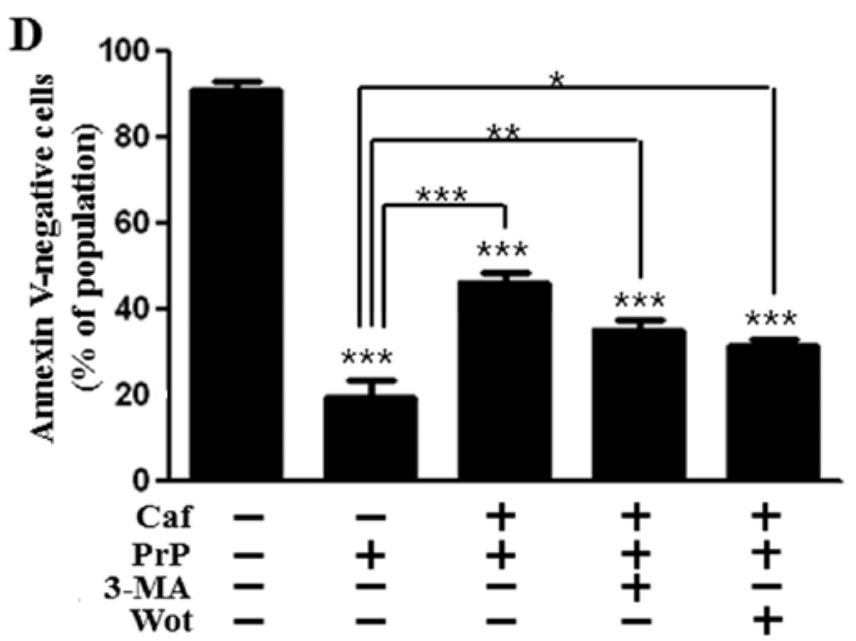

Figure 3. The administration of caffeine inhibits $\operatorname{PrP}(106-126)$-induced apoptosis by activating autophagy. (A) SH-SY5Y cells were pre-treated with the autophagy inhibitors $(1 \mathrm{~h}), 3$-methyladenine (3-MA) $(200 \mu \mathrm{M})$ and wortmannin $(50 \mathrm{nM})$ and were then exposed to caffeine $(8 \mathrm{mM})$ for $5 \mathrm{~h}$. The treated cells were assessed for LC3 production by western blot analysis. Results were normalized to those of $\beta$-actin. (B) The bar graph indicates the average of the LC3-II activity ratio as described in (A). (C) SH-SY5Y cells were exposed to the autophagy inhibitors, 3-MA $(200 \mu \mathrm{M})$ and wortmannin $(50 \mathrm{nM})$. Cell viability was measured by Annexin $\mathrm{V}$ assay. The dots represent fluorescent cells in the plot. Green fluorescence (x-axis) indicates apoptosis and red fluorescence (y-axis) indicates necrosis. Cells in the first quadrant signify the late apoptotic condition and cells in the fourth quadrant signify the early apoptotic condition. (D) The bar graph indicates the average of cell viability measured using an Annexin $\mathrm{V}$ assay. ${ }^{*} \mathrm{P}<0.05,{ }^{* *} \mathrm{P}<0.01$ and ${ }^{* * *} \mathrm{P}<0.001$ : significant differences between the control and each treatment group. Caf, caffeine; Wot, wortmannin; adj.volume, adjustment of volume (band volume minus background volume). 

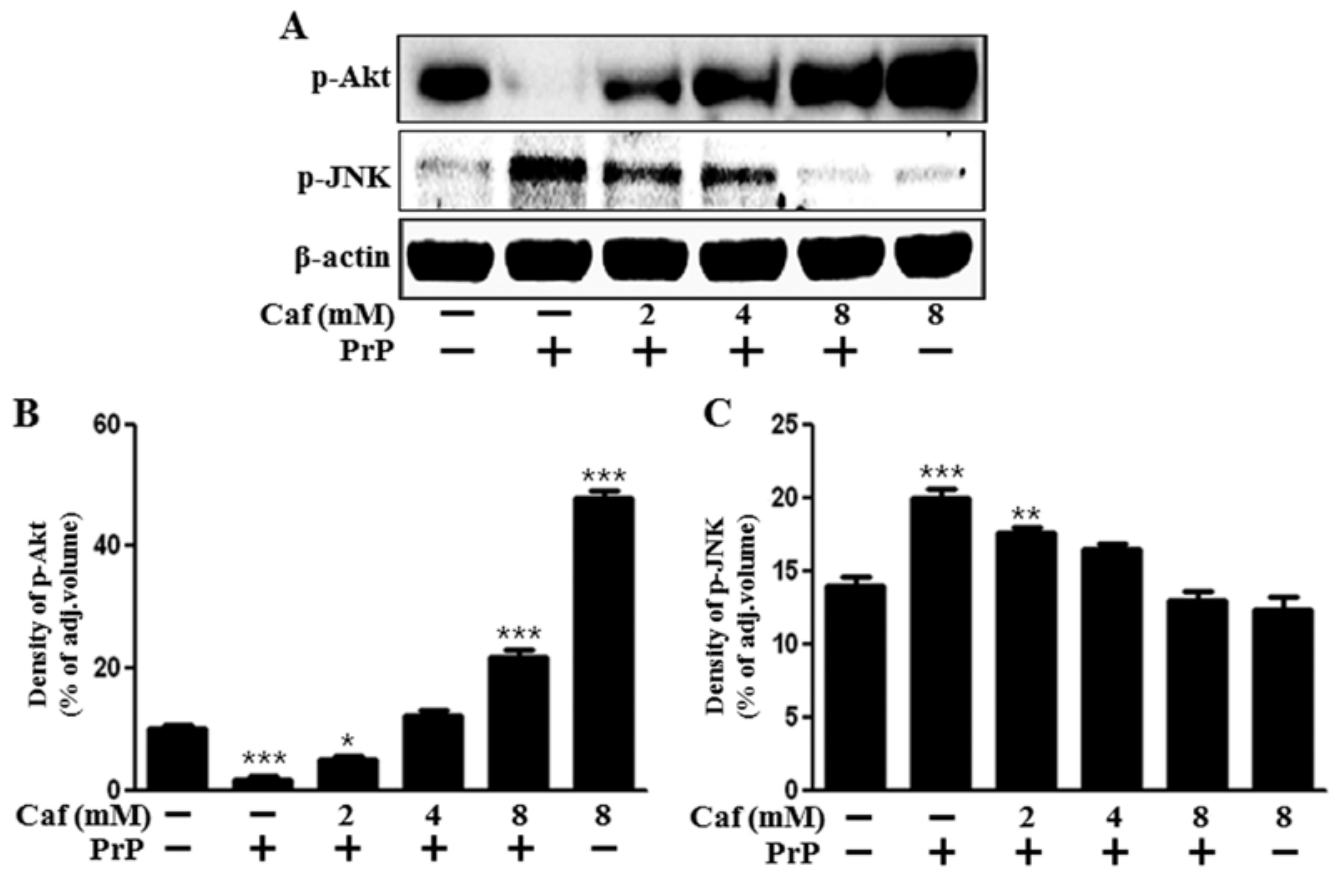

Figure 4. Caffeine mediates survival and death signals. (A) SH-SY5Y cells were treated with various doses of caffeine. The treated cells were assessed for phospho-Akt and phospho-JNK production by western blot analysis. Results were normalized to those of $\beta$-actin. (B) The bar graph indicates the average of the phospho-Akt activity ratio. (C) The bar graph indicates the average of the phospho-JNK activity ratio. ${ }^{*} \mathrm{P}<0.05,{ }^{* *} \mathrm{P}<0.01$ and ${ }^{* * *} \mathrm{P}<0.001$ : significant differences between the control and each treatment group. Caf, caffeine; p-Akt, phosphoryled Akt; p-JNK, phosphoryled JNK; adj. volume, adjustment of volume (band volume minus background volume).

Effects of caffeine on Akt and JNK signaling. We then investigated whether caffeine has an effect on growth and death signals. It is well known that Akt promotes growth factor-mediated cell survival both directly and indirectly. Nakaso et al demonstrated that caffeine prevents the apoptosis of neurons through Akt activation (20). JNK activation has been shown to be induced by $\operatorname{PrP}(106-126)$-induced apoptosis (21). Therefore, we examined Akt and JNK activation following treatment with caffeine and $\operatorname{PrP}(106-126)$. Our results demonstrated that treatment with caffeine reversed the suppressive effects on Akt activation induced by $\operatorname{PrP}(106-126)$ and suppressed the activation of JNK induced by $\operatorname{PrP}(106-126)$. These results suggest that caffeine exerts protective effects on intracellular signaling. Moreover, our resuts suggest that caffeine plays a role similar to that of a JNK inhibitor in cell apoptosis. Based on these results, we suggest that treatment with caffeine inhibits the $\operatorname{PrP}(106-126)$ mediated neuronal apoptotic pathway by regulating autophagy.

\section{Discussion}

The aim of this study was to investigate the role of caffeine-induced autophagy and the regulation of $\operatorname{PrP}(106-126)$ induced apoptosis by caffeine in neuronal cells. Our results suggest that the increase in autophagy induced by caffeine and the resultant reduction in $\operatorname{PrP}(106-126)$-induced neurotoxicity may be the key mechanisms underlying the observed neuroprotective effects of caffeine.

Caffeine is widely used in modern society and is classified as safe by the food and drug administration. Beverages containing caffeine, such as coffee, tea, soft drinks and energy drinks, enjoy great popularity. Caffeine is a psychoactive drug and functions as a central nervous system stimulant, tempo- rarily relieving drowsiness and restoring alertness. A recent study suggested that caffeine induces neuronal GSH synthesis by promoting cysteine uptake, thus leading to neuroprotection (22). Caffeine has also been shown to exert protective effects against Alzheimer's and Parkinson's disease in human and animal studies (23).

Certain studies have suggested that caffeine induces autophagy $(24,25)$. Several other studies have suggested that autophagy is a double-edged sword, with both a beneficial and a harmful potential in cancer (26) and neurodegeneration (27). On the positive side, autophagy is the cellular pathway that mediates the lysosomal degradation of intracellular long-lived macromolecules or organelles for subsequent reuse under physiological conditions of differentiation, starvation, or stress, such as oxidative stress, endoplasmic reticulum stress and the accumulation of abnormal protein $(9,28)$. Saiki et al reported that caffeine at concentrations $>2.5 \mathrm{mM}$ induced apoptosis through the enhancement of autophagy and that the levels of LC3-II were markedly increased at concentrations $>10 \mathrm{mM}(25)$. However, in this study, $8 \mathrm{mM}$ caffeine did not decrease cell viability compared with the control, although it clearly increased the levels of the autophagosomal marker, LC3-II. We also found that caffeine induced apoptosis at concentrations $>10 \mathrm{mM}$ (data not shown). We suggest that the caffeine used in the study by Saiki et al (25) was slightly different from the one we used, with respect to certain characteristics, such as purity or grade. Based on our findings, we suggest that caffeine exerts neuroprotective effects through the induction of autophagy.

Certain studies have shown that caffeine inhibits the PI3K/Akt pathway $(29,30)$. By contrast, our experimental data indicated that Akt activation was increased by treatment with caffeine. Nakaso et al also suggested that caffeine activated the 
PI3K/Akt pathway in SH-SY5Y cells (20). As shown in Fig. 4A and C, the activation of JNK by $\operatorname{PrP}(106-12)$ was decreased by treatment with caffeine. This indicates that caffeine suppresses apoptosis-related signaling in neuronal cells, as JNK is closely associated with apoptosis in neurons (31). These findings support our hypothesis that caffeine exerts protective effects against $\operatorname{PrP}(106-126)$-induced apoptosis in SH-SY5Y cells.

The cross-talk between apoptosis and autophagy is complex and sometimes contradictory; however, it is a critical determinant of the overall fate of the cell. To the best of our knowledge, this is the first study indicating that caffeine-mediated autophagy may play a critical role in the neuroprotection against PrP(106-126)-induced neurotoxicity. We therefore suggest that caffeine may be a valid therapeutic agent for prionrelated neurodegenerative diseases.

\section{Acknowledgements}

This study was supported by a grant from the National Research Foundation of Korea (NRF), funded by the Korean government (MISP) (2013R1A4A1069486).

\section{References}

1. Costanzo M and Zurzolo C: The cell biology of prion-like spread of protein aggregates: mechanisms and implication in neurodegeneration. Biochem J 452: 1-17, 2013.

2. Prusiner SB: Novel proteinaceous infectious particles cause scrapie. Science 216: 136-144, 1982.

3. Prusiner SB: Prions. Proc Natl Acad Sci USA 95: 13363-13383, 1998.

4. Aguzzi A: Staining, straining and restraining prions. Nat Neurosci 11: 1239-1240, 2008.

5. Forloni G, Angeretti N, Chiesa R, et al: Neurotoxicity of a prion protein fragment. Nature 362: 543-546, 1993.

6. Selvaggini C, De Gioia L, Cantu L, et al: Molecular characteristics of a protease-resistant, amyloidogenic and neurotoxic peptide homologous to residues 106-126 of the prion protein. Biochem Biophys Res Commun 194: 1380-1386, 1993.

7. Deter RL and De Duve C: Influence of glucagon, an inducer of cellular autophagy, on some physical properties of rat liver lysosomes. J Cell Biol 33: 437-449, 1967.

8. Rubinsztein DC, Marino G and Kroemer G: Autophagy and aging. Cell 146: 682-695, 2011.

9. Glick D, Barth S and Macleod KF: Autophagy: cellular and molecular mechanisms. J Pathol 221: 3-12, 2010

10. Mizushima N, Yoshimori T and Levine B: Methods in mammalian autophagy research. Cell 140: 313-326, 2010.

11. Levine B and Kroemer G: Autophagy in the pathogenesis of disease. Cell 132: 27-42, 2008.

12. Filomeni G, Graziani I, De Zio D, et al: Neuroprotection of kaempferol by autophagy in models of rotenone-mediated acute toxicity: possible implications for Parkinson's disease. Neurobiol Aging 33: 767-785, 2012.
13. Garcia JJ, Pinol-Ripoll G, Martinez-Ballarin E, et al: Melatonin reduces membrane rigidity and oxidative damage in the brain of SAMP8 mice. Neurobiol Aging 32: 2045-2054, 2011.

14. Nakagaki T, Satoh K, Ishibashi D, et al: FK506 reduces abnormal prion protein through the activation of autolysosomal degradation and prolongs survival in prion-infected mice. Autophagy 9: 2013.

15. Xu K, Xu YH, Chen JF and Schwarzschild MA: Neuroprotection by caffeine: time course and role of its metabolites in the MPTP model of Parkinson's disease. Neuroscience 167: 475-481, 2010.

16. Rubinsztein DC, Cuervo AM, Ravikumar B, et al: In search of an "autophagomometer". Autophagy 5: 585-589, 2009.

17. Park JH, Lee JE, Lee SJ, et al: Potential autophagy enhancers protect against fipronil-induced apoptosis in SH-SY5Y cells. Toxicol Lett 223: 25-34, 2013.

18. Xia DY, Li W, Qian HR, Yao S, Liu JG and Qi XK: Ischemia preconditioning is neuroprotective in a rat cerebral ischemic injury model through autophagy activation and apoptosis inhibition. Braz J Med Biol Res 46: 580-588, 2013.

19. Kim J, Kim TY, Cho KS, Kim HN and Koh JY: Autophagy activation and neuroprotection by progesterone in the G93A-SOD1 transgenic mouse model of amyotrophic lateral sclerosis. Neurobiol Dis 59: 80-85, 2013.

20. Nakaso K, Ito $S$ and Nakashima K: Caffeine activates the PI3K/Akt pathway and prevents apoptotic cell death in a Parkinson's disease model of SH-SY5Y cells. Neurosci Lett 432: 146-150, 2008.

21. Carimalo J, Cronier S, Petit G, et al: Activation of the JNK-c-Jun pathway during the early phase of neuronal apoptosis induced by PrP106-126 and prion infection. Eur J Neurosci 21: 2311-2319, 2005.

22. Aoyama K, Matsumura N, Watabe M, Wang F, Kikuchi-Utsumi K and Nakaki T: Caffeine and uric acid mediate glutathione synthesis for neuroprotection. Neuroscience 181: 206-215, 2011.

23. Chen X, Ghribi O and Geiger JD: Caffeine protects against disruptions of the blood-brain barrier in animal models of Alzheimer's and Parkinson's diseases. J Alzheimers Dis 20 (Suppl 1): S127-S141, 2010.

24. Winter G, Hazan R, Bakalinsky AT and Abeliovich H: Caffeine induces macroautophagy and confers a cytocidal effect on food spoilage yeast in combination with benzoic acid. Autophagy 4 : 28-36, 2008.

25. Saiki S, Sasazawa Y,Imamichi Y, et al: Caffeine induces apoptosis by enhancement of autophagy via PI3K/Akt/mTOR/p70S6K inhibition. Autophagy 7: 176-187, 2011.

26. White E and DiPaola RS: The double-edged sword of autophagy modulation in cancer. Clin Cancer Res 15: 5308-5316, 2009.

27. Wei K, Wang P and Miao CY: A double-edged sword with therapeutic potential: an updated role of autophagy in ischemic cerebral injury. CNS Neurosci Ther 18: 879-886, 2012.

28. Mizushima N: Autophagy: process and function. Genes Dev 21: 2861-2873, 2007.

29. Foukas LC, Daniele N, Ktori C, Anderson KE, Jensen J and Shepherd PR: Direct effects of caffeine and theophylline on p110 delta and other phosphoinositide 3-kinases. Differential effects on lipid kinase and protein kinase activities. J Biol Chem 277: 37124-37130, 2002.

30. Sarkaria JN, Busby EC, Tibbetts RS, et al: Inhibition of ATM and ATR kinase activities by the radiosensitizing agent, caffeine. Cancer Res 59: 4375-4382, 1999.

31. Davis RJ: Signal transduction by the JNK group of MAP kinases. Cell 103: 239-252, 2000. 